\title{
A Review on Sensor Node Placement Techniques in Wireless Sensor Networks
}

\author{
Husna Zainol Abidin ${ }^{*}$, Norashidah Md. Din ${ }^{\#}$, Nurul Asyikin Mohamed Radzi ${ }^{\#}$, Zairi Ismael Rizman ${ }^{\&}$ \\ *Faculty of Electrical Engineering, Universiti Teknologi MARA, 40450 Shah Alam, Selangor, Malaysia \\ E-mail: husnaza@salam.uitm.edu.my

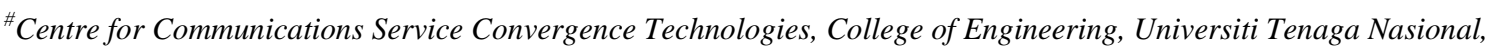 \\ Jalan IKRAM-UNITEN, 43000 Kajang, Selangor, Malaysia \\ E-mail:norashidah@uniten.edu.my,asyikin@uniten.edu.my \\ ${ }^{\&}$ Faculty of Electrical Engineering, Universiti Teknologi MARA, 23000 Dungun, Terengganu, Malaysia \\ E-mail: zairi576@tganu.uitm.edu.my
}

\begin{abstract}
One way to provide Wireless Sensor Network (WSN) with maximum coverage, maximum connectivity, minimum deployment cost and minimum energy consumption is through an effective planning mechanism in arranging an optimum number of sensor nodes. Proper planning will provide a cost-effective deployment by having optimal placements for the sensor nodes. Sensor node placement schemes are needed to accommodate the balance of coverage and energy consumption since closer sensor nodes not only reduces the energy consumption but will result in the network coverage becoming smaller. This paper critically reviews the research and development work done in sensor node placement. Based on the review, the design objectives that need to be considered are identified. Most of the work reviewed focused on two or three design objectives.
\end{abstract}

Keywords - sensor node placement; coverage; connectivity; energy consumption

\section{INTRODUCTION}

The advancement in wireless communications and electronics has led to the development of Wireless Sensor Network (WSN). WSN consists of a group of sensor nodes that are able to communicate with each other through wireless medium and have at least a sink node where the data will be sent to. WSN fulfils two fundamental functions that are sensing and communicating with different types of sensors [1]. Thus, it allows an administrator to automatically and remotely monitor any activity precisely. Each sensor node is able to detect any intended event or object, process the detected data, transmit the data and store the data. WSN was originally motivated by military applications such as battlefield surveillance [2], but nowadays it is extensively employed in many applications. One of the crucial problems in implementing a WSN is the position of the nodes that will meet the design specifications such as coverage, connectivity, and energy consumption [3].

Coverage which is affected by the sensor node sensing range is an important issue in WSN that ensures the monitoring area is covered by at least one sensor node and the accuracy of the information depends on the quality of coverage within the sensing range [3]. The coverage itself without full connectivity will deteriorate the quality of WSN. Thus, coverage and connectivity must be considered simultaneously when deploying a WSN [4]. The desired event or object is detected by a sensor node if it is located within the sensing range of the sensor node and this information will be shared with the neighbouring sensor nodes that are located within the sensor node communication range. Therefore, in order to ensure an effective detection in a network and sensor node to communicate with each other, the covered area should be expanded in order to guarantee connectivity [5]. Connectivity which is affected by a sensor node communication range is required to ensure that every sensor node is directly connected to the sink node or indirectly connected via other nodes on multi-hop path connectivity [3] since the communication range is often smaller than the monitoring area size where direct links are impossible.

Energy consumption determines the lifetime or the replacement cycle of the sensor nodes, where it should be minimized to lengthen the lifetime of the sensor nodes [3]. This is because it is uneconomical and not environmental friendly to keep on changing the battery. The intermittent in 
the transmission of data will also occur if some of the nodes die due to no power supply. Hence, data needs to be transmitted again that will cause delay and packet drops. There are three different approaches to conserving energy in WSNs without sacrificing the coverage [6]. The first approach is through coverage deployment strategy, while the second approach is the scheduling of active and sleep sensor nodes modes. The final approach is through the sensing range adjustment. This paper is focusing on the works done on coverage deployment strategy via the optimum sensor node placement. Section II introduces WSN node placement specifically on sensor node placement. A critical review of sensor node placement works is described in Section III, and finally, the whole paper is summarized in Section IV.

\section{MATERIAL AND METHOD}

\section{A. Node Placement}

Generally, the purpose of optimizing node placement is to minimize the energy usage without jeopardizing the required network coverage while the connectivity is guaranteed. The main objective is to find the location to place an appropriate number of nodes so that the coverage is maximized and the connectivity for each sensor node to the sink node is kept significant. There are four types of nodes in WSN known as sensor node, relay node, cluster head and sink node [7]. Sensor node detects the intended event while relay node transmits the detected data to the sink node. The cluster head is responsible for collecting and transferring data in a clustered WSN. The node placement optimization investigated in this paper is only limited to the sensor nodes. Fig. 1 illustrates the node placement strategies in WSN.

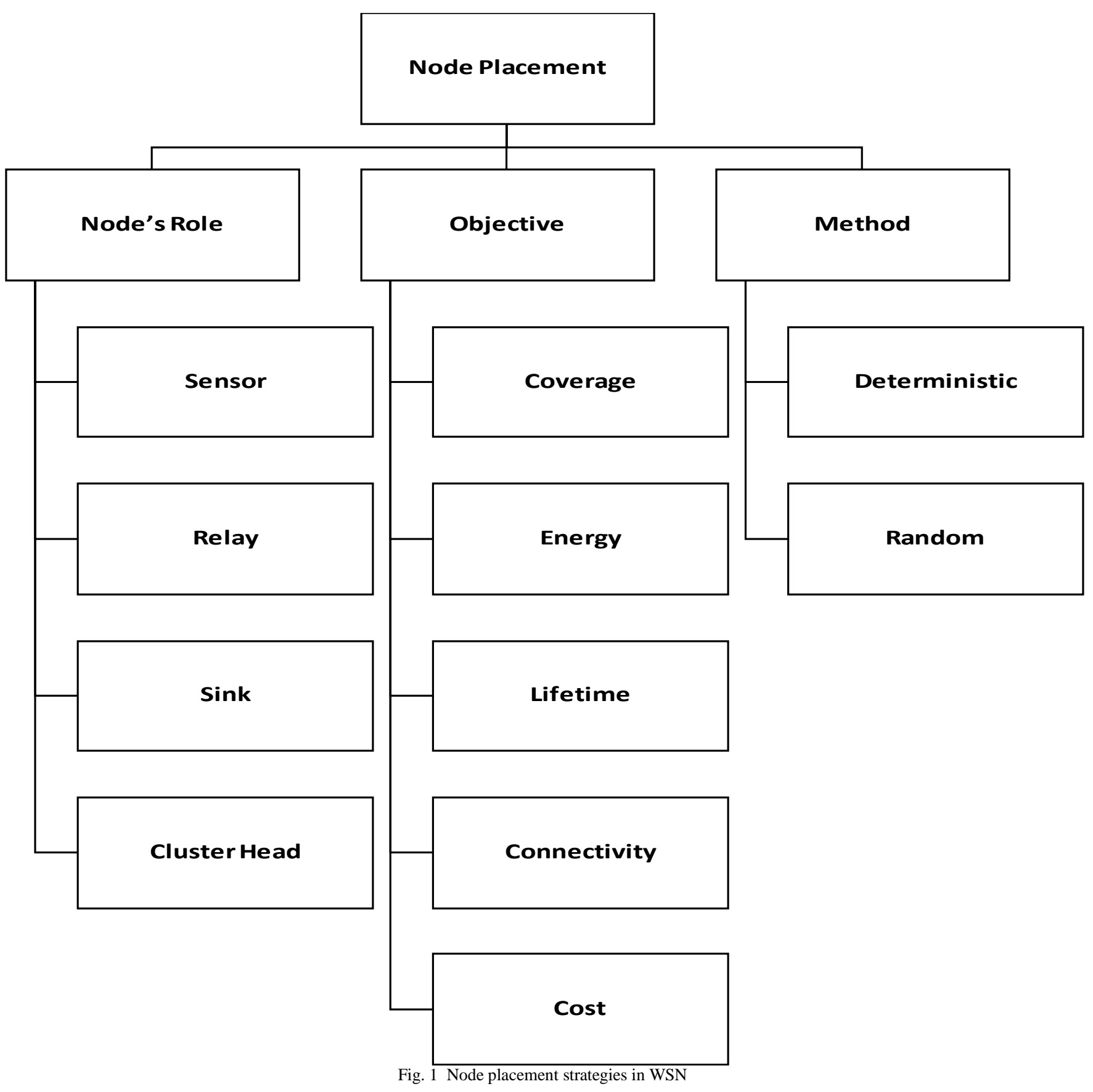




\section{B. Sensor Node Placement}

The positioning of sensor nodes becomes one of the major concerns because the success of WSN operation highly depends on the sensor nodes' position [1]. In [2] have listed key design objectives in deploying sensor nodes which include coverage, energy consumption, a lifetime of a network, connectivity and cost that is represented by the number of sensor nodes. The Quality of Service (QoS) and the economic cost of the network are intercorrelated [1] because an efficient deployment of sensor node will also reduce the construction and communication cost of the network as well as improve the resource management [8]. This is because the optimal sensor node placement enables the administrator to minimize manpower and time to acquire accurate information on monitoring area [8]. The placement optimality criteria used varies depending on the aims and applications of WSN. A common objective is to minimize the number of required sensor nodes in order to cover the whole monitoring area. Minimizing the number of sensor nodes can take the form of an "art gallery" problem in finding the minimum set of locations for security guards inside an art gallery [7]. The boundary of the entire gallery is visible by at least one of the security guards. However, in the art gallery problem, all security guards are assumed to have an infinite vision if there are no obstacles. This assumption is not applicable to WSN since sensor node has limited sensing range.

Sensor nodes are generally battery-operated, and in some cases, energy can be obtained from external resources. However, external power supply sources often exhibit a noncontinuous behaviour [6]. Thus, it is very crucial to find a way to reduce the energy consumption because it is inconvenient to keep on changing the battery especially if it is installed in remote areas. Although the communication methods and protocols of the sensor node may affect the coverage, connectivity, and energy consumption, they are only considered after the sensor node positions have been determined [3]. The energy consumption and the coverage are two conflicting objectives [9]. Bigger coverage is achieved if the distance between two sensor nodes is further. However, their energy consumption will be higher due to longer distance data transmission. Connectivity is similarly critical for WSN because it determines the effective transmission of data as collected data needs to be sent to the sink node [9]. Coverage and connectivity can be optimized by deploying a large number of sensor nodes [3], but the aim of this paper is to guarantee a maximum coverage and connectivity using fewer sensor nodes.

Sensor nodes can be deterministically or randomly deployed depending on the type of sensor node, application and the environment that the sensor nodes will operate in [10]. Deterministic sensor node deployment is more preferable when the monitoring area is physically accessible and necessary when sensor nodes are expensive or when their operation is significantly affected by their locations. Desired coverage can be assured with deterministic deployment since the locations of sensor nodes are carefully planned. Moreover, inefficient patterns can also be avoided, and it is easier for further network expansion.

In contrast, random distribution of sensor nodes is typically used when the monitoring area is physically inaccessible. Although random deployment is cost savvy, maximum coverage may be achieved with higher number of sensor nodes [11] because the desired coverage and accuracy might not be achievable with random deployment [7]. Furthermore, randomly deployed sensor nodes may result in an uneven density of sensor nodes where some areas are densely deployed while the other areas have a low density deployment and may even have no sensor nodes [12]. Denser deployment increases the hardware cost, while a sparse deployment results to coverage holes. Randomly deployed sensor nodes also would cause redundant nodes, which is not cost effective [12]. Thus, it can be said that with random deployment, there is no guarantee for full coverage with an economical hardware cost.

\section{RESULTS AND DISCUSSION}

\section{A. Related Works on Sensor Node Placement}

The problem in finding the optimized position of sensor nodes in a WSN has attracted research and development work looking into the mechanisms for the sensor node placement. Fig. 2 portraits sensor node placement algorithms that are reviewed in this paper. As depicted in the figure, sensor node placement can be divided into two types; dynamic placement and static placement. Dynamic placement involves both homogeneous and heterogeneous sensor nodes while static placement involves homogeneous WSN where only stationary sensor nodes are used. The taxonomy of the algorithms is further outlined in Table 1 and the following subsections review the related work on dynamic and static sensor node placements respectively.

TABLE I

Summary of THe SENSOR Node Placement TAXonomy Features

\begin{tabular}{|l|l|l|l|}
\hline Features & Sensor Node & Method & Type \\
\hline Virtual Force (VF) & Mobile & Repulsive/Attractive Forces & Dynamic \\
\hline Cellular Automata & Mobile & Repulsive/Attractive Forces & Dynamic \\
\hline Centralized and Distributed & Mobile & Heuristic and Self Organized & Dynamic \\
\hline Artificial Intelligence (AI) & Mobile/ Stationary & Optimize & Dynamic/Static \\
\hline Strip Based & Stationary & Sequence & Static \\
\hline Grid & Stationary & Evaluate Discretized Area & Static \\
\hline Modeling & Stationary & Archimedes & Static \\
\hline Search & Stationary & Deployment & Static \\
\hline
\end{tabular}




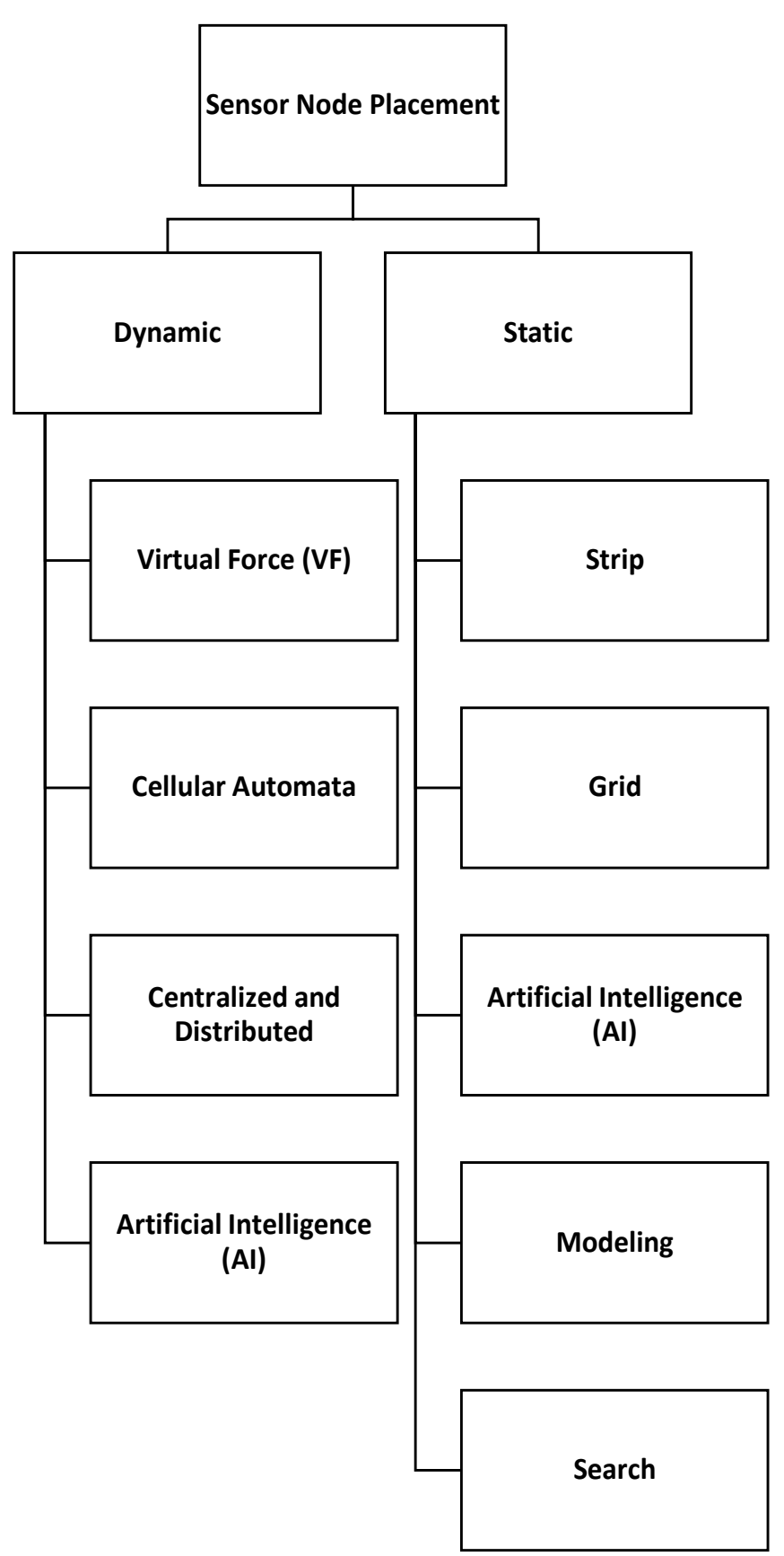

Fig. 2 A taxonomy of sensor node placement algorithms

\section{A. Dynamic Placement}

Dynamic placement in a homogeneous WSN utilizes only mobile sensor nodes while heterogeneous WSN uses both stationary and mobile sensor nodes. Stationary sensor nodes can be deterministically deployed or randomly deployed. Normally, it is chosen due to energy saving and to reduce costs. However, the main setback is after sensor nodes' first positioning; there will not be any further mobility in the network. Thus, optimal locations can be found by using an offline scheme as a facility location optimization problem [5]. In dynamic sensor node placement, mobile sensor node that has a mobility feature is used to fill up the coverage holes in randomly deployed sensor nodes.

In a heterogeneous WSN, stationary sensor nodes are initially deployed while mobile sensor nodes will move to the areas that are not covered by the stationary sensor nodes. Mobile sensor nodes are normally added after the initial deployment of stationary sensor nodes to solve the coverage hole problem occurs due to uneven sensor nodes distribution. In a homogeneous WSN with mobile sensor nodes, only mobile sensor nodes are deployed randomly. These sensor nodes will move around the monitoring area to fulfil the design objectives. Although mobile sensor node is believed to be able to address the coverage hole problem, there will be additional hardware [36] costs for mobility supports, and it also consumes more energy due to mobility [4].

Virtual Force (VF) is an algorithm that is used for mobile sensor node placement. It is believed that VF based algorithm works well for WSN that consists of mobile sensor nodes only [5] because the performance of WSN will be degraded if VF is deployed in a heterogeneous WSN due to virtual forces emitted by the stationary sensor nodes [13]. VF uses the repulsive and attractive forces in order to ensure sensor nodes are distributed evenly. The sensor nodes, the obstacles, and the monitoring areas are modelled as points subject to attractive or repulsive force between them [14]. In [14] proposes a VF based node placement algorithm using a force model based on Van Der Waals force. However, this work only considers coverage rate without considering energy consumption and connectivity.

Cellular automata based algorithms are applied in [3], [15]. A cellular automaton is a system that consists of a large number of simple processing elements or cells that interact among themselves [3]. In [15] stated that cellular automata based algorithms are robust against any inaccuracies that might happen during measurement of sensor node positions or in the movement of sensor nodes. Similarly to VF, this algorithm only suitable to be deployed in homogeneous WSN with mobile sensor nodes because it deals with the repulsive and attractive forces. This work, however, did not consider any connectivity and energy consumption requirement.

In [3] suggest cellular automata based algorithm to achieve adequate coverage, connectivity, and sparsity that would be able to stand with any environmental changes. However, this algorithm requires more nodes than optimal deterministic placement in order to achieve desired redundancy and more sensor node movement than what is necessary. This may significantly reduce the lifetime of the network by depleting the sensor nodes energy if it is not properly managed.

In [16] propose a centralized and distributed algorithm movement. The objective is to deploy the least number of sensor nodes, and the maximum displacement is minimized. Hence, energy consumption will be reduced.

Most researchers nowadays prefer the Artificial Intelligence (AI) approaches based on biologically inspired algorithms for node placement in WSN. An Evolutionary Approach based on Voronoi Diagram (EAVD) sensor node placement algorithm is introduced in references [17]. In EAVD, stationary sensor nodes are randomly deployed then the area is divided into Voronoi cells. Genetic Algorithm (GA) is then used to deploy additional mobile sensor nodes in each cell to heal coverage holes. However, maximum connectivity and deployment cost are not considered in this work.

A Genetic Algorithm and Voronoi Vertex Averaging Algorithm (VVAA) is proposed in [12] to relocate the 
mobile sensor nodes in addressing the coverage holes problem. GA is used to find the optimum locations while coverage holes are detected by using Voronoi Diagram. Although the algorithm offers the highest throughput, but the connectivity cannot be said $100 \%$ guaranteed due to high packet loss. The energy consumption of this algorithm also is higher compared to GA alone and random deployment. It can be concluded that this work only considers coverage of the WSN without considering connectivity and energy [35] consumption of the network.

Sensor node placement algorithm based on Glowworm Swarm Optimization (GSO ) is introduced by [18]. The aim of this work is to maximize the coverage for a fixed number of mobile sensor nodes. This work is inspired by the behaviour of a glowworm that carries a luminescent substance called luciferin. The movement of a glowworm is decided by the intensity of luciferin possessed by its neighbours. Each glowworm will be attracted towards the brighter glow of other glowworms in the neighbourhood and it will move towards the brightest neighbour. GSO is applied to solve the sensor node placement where each sensor node is treated as an individual glowworm. However, in this case, the sensor node will be moving towards its neighbour that has the lower intensity of the luciferin which is depending on the distance from their neighbouring sensor nodes. Sensor nodes receive luminance from its neighbouring sensor nodes situated within the communication range. It means sensor node will move towards its dimmer sensor node which is beyond the communication range to ensure that the distance between the two sensor nodes will become closer. The idea of repulsion and attraction is more or less similar to VF. Thus this algorithm is more suitable for arranging mobile sensor nodes. Furthermore, this algorithm is referring to the communication range for coverage, but theoretically, in order to achieve maximum coverage, the algorithm should be dealing with the sensing range. Communication range is normally associated with connectivity. Another setback of this algorithm is that it is only focusing on coverage and energy consumption which is related to the total moving distance without considering any connectivity requirement.

Artificial Bee Colony (ABC) optimization for sensor node placement is suggested in reference [5]. ABC was developed based on the foraging behaviour of a honey bee swarm. The objective of this work is to rearrange mobile sensor nodes in a homogeneous WSN that will maximize the coverage rate of the network. Nevertheless, this work only considers coverage and do not consider energy consumption and connectivity.

In [19] utilized a new optimization technique called Territorial Predator Scent Marking Algorithm (TPSMA) to redeploy mobile sensor nodes. TPSMA imitates the behaviour of a territorial predator in marking their territories with their odours. Two objectives were focused on this work which are the maximum coverage and minimum movement. Mobile sensor nodes movement are minimized in order to reduce the energy consumption due to sensor nodes mobility. To ensure full connectivity, the distance from a sensor node to another must be within the communication range to ensure that full connectivity can be achieved via multi-hop path connectivity. Only one sensor node can be placed at a grid to minimize the cost.

\section{B. Static Placement}

Various algorithms have been developed for static placement such as grid architecture, searching, modelling, strip based and AI. Grid architecture divides the monitoring area into a number of small grids where a grid is considered covered if it is located within the sensing range of at least a sensor node. Grid calibration for sensor node placement is proposed by [20] to ensure that all grids are covered by sensor nodes. Initially, sensor nodes are placed at all grid points. Then, each sensor node will be removed. If the grid is still covered by a sensor node even though another sensor node is removed, that point is considered does not need a sensor node. Grid calibration algorithm done by [20] is carried out based on the energy consumption distribution. The area which has higher energy consumption needs more sensor nodes. Hence, the energy hole can be avoided. However, this work is not suitable for predeployment because the energy consumed at particular area needs to be determined first. This work also does not consider connectivity, and the number of required sensor nodes is considered high.

In [21] discuss three placement strategies known as the grid, triangular and strip to in a WSN used for detecting smoke. The aim is to ensure maximum network lifetime and maximum coverage with a minimum number of sensor nodes. Based on their analysis, there is no significant difference among the three strategies in terms of lifetime. However, grid deployment scheme is the most cost effective that can provide maximum coverage with the least number of sensor nodes.

In [22] suggest the use of a searching algorithm known as Oppositional Gravitational Search Algorithm (OGSA) to provide maximum coverage with guaranteed connectivity. This algorithm accomplishes the $l$-coverage and $n$ connectivity of the sensor nodes in order to ascertain the minimum number of preferred possible locations to place the sensor nodes. The method uses agent representation, derivation of efficient fitness function as well as the usual Gravitational Search algorithm operators.

In [23] utilize a modelling method known as Archimedes' spiral modelling to solve the unbalanced energy consumption which will then prolong the network lifetime. Based on their observation, the proposed algorithm performs better than the other two algorithms in terms of network lifetime as well as the throughput and end to end delay. However, this result does not indicate whether the algorithm can give maximum coverage with maximum connectivity or not. The throughput and end-to-end delay might come from certain sensor nodes only and does not show that it is a fully connected WSN.

A strip based algorithm was presented by [24] to ensure that any point inside a belt area is covered by at least two sensor nodes. The proposed algorithm is based on the Equalization Strip Theory and the Isosceles Triangle strip pattern. The proposed algorithm is capable of offering considerable maximum coverage with least number of sensor nodes. Nevertheless, there is no report on connectivity and energy consumption.

The vast application of GA based algorithms is mostly used in deterministic sensor node placement. However, GA prone to premature convergence phenomenon and difficult to 
ensure accuracy [25] because it starts from a population of random solutions then recombines and mutates them with the aim to be improved without taking into account the specificity of the problem [1].

In [11], coverage capability is used to measure the WSN deployment where it refers to the amount of information captured by the WSN. Based on the limited sensing range of each sensor node and the limited number of sensor nodes, the sensor nodes are deployed where the most information can be captured. However, the coverage quality is based on the amount of information captured which cannot be predetermined. This algorithm also did not consider connectivity and energy consumption.

In [8] address the problem of maximizing coverage and minimizing the number of sensor nodes. The issue of area coverage, where the number of sensor nodes is reduced to cover the monitoring area is resolved by using this algorithm. This is done by altering the number of sensor nodes in a binary-search routine, where it is functional for other types of coverage problems as well. However, the drawback of this algorithm is they do not consider connectivity and the energy consumption.

An Evolutionary Algorithm (EA) is utilized in [26] sensor node placement in an environmental monitoring system known as Environmental Sensor Networks (ESN). This is to ensure data quality by ensuring the robustness of the network when some sensor nodes fail through sensor nodes redundancy. However, it is not reported whether this algorithm is able to provide full connectivity which is important in providing data quality delivery. Other developed bioinspired based sensor node placement algorithms are Ant Colony Optimization (ACO) and TPSMA.

In [1] propose ACO to optimize the number of sensor nodes while the constraints are full coverage and full connectivity. A non-fixed amount of sensor nodes is placed in the monitoring area, but it must be positioned so that it is fully covered and fully connected with a minimum number of sensor nodes. ACO is seen to be more accurate than ACO starts at the beginning of the trail. With full coverage and full connectivity constraints specified in this work, WSN with full coverage and full connectivity is guaranteed, but the work did not consider energy consumption.

TPSMA with two single objective approaches was implemented by [27], [28] which were focusing on the maximum coverage and minimum energy consumption. In order to ensure full connectivity, the distance between the two sensor nodes must be within the communication range, and there must be at least a path from each sensor node to the sink node via multi-hop routing or direct routing. The number of sensor nodes deployed is also kept to a minimum to reduce the deployment cost.

\section{Multi-Objective Optimization}

In many real life problems, there are several contradicting objectives that need to be optimized simultaneously [29]. Thus, it should not depend on a single optimal solution, but possible solutions of equivalent quality. The sensor node placement problem also falls in this category. In [2] has discussed other trade-offs effects affected by the distance between sensor nodes. Maximizing coverage means more sensor nodes are placed far apart from the sink node because it supposed to be placed closer to sink node to reduce the energy consumption. If the number of sensor nodes increases, net energy consumption will also increase, but the energy consumed by each node will be reduced. This shows that both objectives are conflicting. Hence, it is believed that a Multi-Objectives Optimization (MOO) approach is suitable for a problem with multiple and conflicting objectives. None of the AI based works presented earlier consider MOO.

In [9] propose a mobile sensor node placement algorithm that is based on multi-objective PSO (MOPSO) with fuzzy logic based strategy to select the best compromised solution on the Pareto front. MOPSO with fuzzy logic is also proposed by [30], where three main objectives are passed on to a Fuzzy Logic Engine to form the fuzzy rules. The three objectives that are considered in this work are coverage, connectivity and network lifetime. Fuzzy logic is known to give higher accuracy result for decision making, but it has a setback in terms of execution time. The only aspect that is not considered in this work is deployment cost.

Swarm Intelligence (SI) which is based on information sharing of PSO and maintenance mechanism of an Artificial Immune System (AIS) is proposed to address coverage problems [31]. The problem considers the influence of communication range, sensing range and the coverage probability of sensor nodes due to the different conditions in different sections in a real environment. This work is considered as a MOO approach because their main objectives are to obtain maximum coverage with given number of sensor nodes and a minimum number of sensor nodes with required coverage probability. However, the objectives of this algorithm are not executed concurrently, where the output for each objective will be different. Thus, there will be two sets of sensor node coordinates to be produced. Moreover, it does not guarantee connectivity and minimum energy consumption.

Recent works show that EA has been enhanced to deal with MOO problems. Multi-Objective Evolutionary Algorithms (MOEAs) aim at finding a set of representative Pareto optimal solution in a single run. Most MOEAs are using Pareto dominance, where single objective EA frameworks are adopted, and the fitness of each solution at each generation is determined by its Pareto dominance relations with other solutions in the population. In [32] believed that MOEA is able to improve convergence speed and algorithm efficiency.

In [2], [32] propose sensor node placement algorithms that utilize MOEA approach with decomposition and Pareto optimal concepts. Decomposition method is able to speed up the evaluation process because the evaluation for all objectives is carried out concurrently. MOEA approach by [32] considers network size, power consumption and processing time as their main objectives without considering any coverage and connectivity requirement which are more important for WSN. Alternatively, in [2] suggested MOEA which focuses on coverage, lifetime, energy consumption as well as the connectivity. However, this algorithm does not limit any number of deployed sensor nodes which would affect the cost and total energy consumption.

A multi-objective ACO algorithm is proposed in [33] for lunar surveying to ensure maximum coverage with minimum energy consumption. The proposed algorithm is carried out 
with Pareto frontier. Based on their observation, it is found that the proposed algorithm outperforms Four-Directional Placement (FDP) with maximum coverage and minimum energy consumption.

A Multi Objective TPSMA (MOTPSMA) is developed by [34] which is the extension of TPSMA presented in the previous subsection. In MOTPSMA, both objective functions are executed concurrently using Pareto dominance and decomposition technique with similar constraints on full connectivity and minimum deployment cost.

The reviewed sensor node placement algorithms are tabulated in Table 2. It can be concluded that most of these algorithms intend to arrange the sensor nodes that would be able to give maximum coverage with lower energy usage. Other important aspects that are considered includes connectivity and deployment cost.

TABLE II

REVIEW ON SENSOR NODE PlaCEMENT ALGORITHMS

\begin{tabular}{|c|c|c|c|c|}
\hline \multirow{2}{*}{ Algorithm } & \multicolumn{4}{|c|}{ Objective } \\
\hline & Coverage & Connectivity & Energy & Cost \\
\hline Van Der Waals force [14] & $\sqrt{ }$ & & & \\
\hline Cellular Automata [3] & $\sqrt{ }$ & $\sqrt{ }$ & & \\
\hline Cellular Automata [15] & $\sqrt{ }$ & & & \\
\hline Centralized and distributed movement [16] & $\sqrt{ }$ & & $\sqrt{ }$ & $\sqrt{ }$ \\
\hline EAVD [17] & $\sqrt{ }$ & & & \\
\hline VVAA [12] & $\sqrt{ }$ & & & \\
\hline GSO [18] & $\sqrt{ }$ & & $\sqrt{ }$ & \\
\hline $\mathrm{ABC}[5]$ & $\sqrt{ }$ & & & \\
\hline TPSMA [19] & $\sqrt{1}$ & & $\sqrt{ }$ & \\
\hline Grid calibration [20] & $\sqrt{ }$ & & $\sqrt{ }$ & \\
\hline Grid, triagular and strip [21] & $\sqrt{ }$ & & $\sqrt{ }$ & $\sqrt{ }$ \\
\hline OGSA [22] & $\sqrt{ }$ & $\sqrt{ }$ & & \\
\hline Archimedes' Spiral Modeling [23] & & & $\sqrt{ }$ & \\
\hline Strip based [24] & $\sqrt{ }$ & & & $\sqrt{ }$ \\
\hline OPEN [11] & $\sqrt{ }$ & & & \\
\hline GA [8] & $\sqrt{ }$ & & & $\sqrt{ }$ \\
\hline EA [26] & & $\sqrt{ }$ & & \\
\hline $\mathrm{ACO}[1]$ & $\sqrt{ }$ & $\sqrt{ }$ & & \\
\hline TPSMA [27], [28] & $\sqrt{ }$ & $\sqrt{ }$ & $\sqrt{ }$ & $\sqrt{ }$ \\
\hline MOPSO and Fuzzy Logic [9] & $\sqrt{ }$ & $\sqrt{ }$ & $\sqrt{ }$ & \\
\hline MOPSO and Fuzzy Logic [30] & $\sqrt{ }$ & $\sqrt{ }$ & $\sqrt{ }$ & \\
\hline SI [31] & $\sqrt{1}$ & & & $\sqrt{ }$ \\
\hline Decomposition Pareto dominance MOEA [32] & & & $\sqrt{ }$ & $\sqrt{ }$ \\
\hline Decomposition Pareto dominance MOEA [2] & $\sqrt{ }$ & $\sqrt{ }$ & $\sqrt{ }$ & \\
\hline MOACO [33] & $\sqrt{ }$ & & $\sqrt{ }$ & \\
\hline MOTPSMA [34] & $\sqrt{ }$ & $\sqrt{ }$ & $\sqrt{ }$ & $\sqrt{ }$ \\
\hline
\end{tabular}

\section{CONCLUSION}

This paper reviewed works done on sensor node placement algorithms. The arrangement of sensor nodes is very important in order to achieve a maximum coverage with minimum energy consumption without jeopardizing the connectivity and the deployment cost. There is a tradeoff among these attributes which need to be considered when arranging the sensor nodes. Thus, an effective algorithm is needed to ensure that these objectives can be achieved. It can be seen the trend shows that AI based algorithms are mostly being used nowadays either on its own or hybrid with another algorithm due to its specialty mentioned above. The sensor node placement problem is also considered as a MOO problem due to the tradeoff mentioned before. Hence, MOO based algorithms are proposed by some researchers. The works described in this paper do not consider all important aspects of arranging the sensor nodes such as coverage, energy consumption, connectivity and deployment cost concurrently. Most of them only focused on coverage and energy consumption.

\section{ACKNOWLEDGMENT}

This research is supported by the Ministry of Higher Education (MOHE) Malaysia under the Fundamental Research Grant Scheme (FRGS) (File Number: FRGS/1/2015/ICT03/UITM/02/4).

\section{REFERENCES}

[1] S. Fidanova, P. Marinov, and E. Alba, Ant Algorithm for Optimal Sensor Deployment, ser. Computational Intelligence, 2012, vol. 399.

[2] S. Sengupta, S. Das, M. D. Nasir, and B. K. Panigrahi, "Multiobjective node deployment in WSNs: In search of an optimal tradeoff among coverage, lifetime, energy consumption, and connectivity," Engineering Applications of Artificial Intelligence, vol. 26, pp. 405-416, Jan. 2013.

[3] S. Torbey and S. G. Akl, "Reliable node placement in wireless sensor networks using cellular automata," in Proc. ICUCNC'12, 2012, p. 210.

[4] S. L. Cheng, L. C. Yang, and Y. C. Sing, "The divide-and-conquer deployment algorithm based on triangles for wireless sensor networks," IEEE Sensors Journal, vol. 11, pp. 781-790, Mar. 2011.

[5] C. Ozturk, D. Karaboga, and B. Gorkemli, "Artificial bee colony algorithm for dynamic deployment of wireless sensor networks," Turkish Journal of Electrical Engineering and Computer Sciences, 
vol. 20, pp. 255-262, Feb. 2012.

[6] C. Zhu, C. Zheng, L. Shu, and G. Han, "A survey on coverage and connectivity issues in wireless sensor networks," Journal of Network and Computer Applications, vol. 35, pp. 619-632, Mar. 2012.

[7] M. Younis and K. Akkaya, "Strategies and techniques for node placement in wireless sensor networks: A survey," Ad Hoc Networks, vol. 6, pp. 621-655, Jun. 2008.

[8] Y. Yoon and Y. H. Kim, "An efficient genetic algorithm for maximum coverage deployment in wireless sensor networks," IEEE Transactions on Cybernetics, vol. 43, pp. 1473-1483, Oct. 2013.

[9] P. M. Pradhan and G. Panda, "Connectivity constrained wireless sensor deployment using multiobjective evolutionary algorithms and fuzzy decision making," Ad Hoc Networks, vol. 10, pp. 1134-1145, Aug. 2012.

[10] B. Krishnamachari, Networking Wireless Sensors. Cambridge, England: Cambridge University Press, 2011.

[11] L. Zhang, D. Li, H. Zhu, and L. Cui, "OPEN: An optimisation scheme of $\mathrm{N}$-node coverage in wireless sensor networks," IET Wireless Sensor Systems, vol. 2, pp. 40-51, Mar. 2012.

[12] V. V. Juli and J. Raja, "Mobility assisted optimization algorithms for sensor node deployment," European Journal of Scientific Research, vol. 78, pp. 156-167, 2012.

[13] X. Wang and S. Wang, "Hierarchical deployment optimization for wireless sensor networks," IEEE Transactions on Mobile Computing, vol. 10, pp. 1028-1041, Jul. 2011.

[14] X. Yu, N. Liu, W. Huang, X. Qian, and T. Zhang, "A node deployment algorithm based on van der Waals force in wireless sensor networks," International Journal of Distributed Sensor Networks, vol. 2013, pp. 1-9, Oct. 2013.

[15] M. Esnaashari and M. R. Meybodi, "Deployment of a mobile wireless sensor network with k-coverage constraint: A cellular learning automata approach," Wireless Networks, vol. 19, pp. 945968, Jul. 2013.

[16] D. Saha and N. Das, "Self-organized area coverage in wireless sensor networks by limited node mobility," Innovations in Systems and Software Engineering, vol. 12, pp. 227-238, Sep. 2016.

[17] N. Rahmani and F. Nematy, "EAVD: An evolutionary approach based on Voronoi diagram for node deployment in wireless sensor networks," in Proc. SocProS'11, 2012, p. 121.

[18] W. H. Liao, Y. C. Kao, and Y. S. Li, "A sensor deployment approach using glowworm swarm optimization algorithm in wireless sensor networks," Expert Systems with Applications, vol. 38, pp. 1218012188, Sep. 2011.

[19] H. Z. Abidin, N. M. Din, and N. A. M. Radzi, "TPSMA based sensor node redeployment for mobile wireless sensor networks," in Proc. $A C C N^{\prime} 15,2015$, p. 78.

[20] C. Tang, Y. Cheng, and J. Yin, "An optimized algorithm of grid calibration in WSN node deployment based on the energy consumption," Journal of Information and Computational Science, vol. 9, pp. 1035-1042, 2012 .

[21] M. Maksimovic and V. Milosevic, "Evaluating the optimal sensor placement for smoke detection," Yugoslav Journal of Operations Research, vol. 26, pp. 33-50, Jan. 2016.

[22] C. Jehan and D. S. Punithavathani, "Potential position node placement approach via oppositional gravitational search for fulfill coverage and connectivity in target based wireless sensor networks,"
Wireless Networks, pp. 1-14, 2016.

[23] S. Halder and S. D. Bit, "Design of an Archimedes' spiral based node deployment scheme targeting enhancement of network lifetime in wireless sensor networks," Journal of Network and Computer Applications, vol. 47, pp. 147-167, Jan. 2015.

[24] T. Wang, Y. Sun, Z. Xu, W. Zhai, and R. Zhuo, "Node placement for long bounded belt complete 2-coverage in wireless sensor networks," Mathematical Problems in Engineering, vol. 2015, pp. 1-13, Nov. 2015 .

[25] J. Zhao and H. Sun, "Intelligent single particle optimizer based wireless sensor networks adaptive coverage," Journal of Convergence Information Technology, vol. 7, pp. 153-159, Feb. 2012

[26] S. Budi, P. de Souza, G. Timms, V. Malhotra, and P. Turner, "Optimisation in the design of environmental sensor networks with robustness consideration," Sensors, vol. 15, pp. 29675-29781, Nov. 2015.

[27] H. Z. Abidin and N. M. Din, "Sensor node placement in wireless sensor network based on territorial predator scent marking algorithm," ISRN Sensor Networks, vol. 2013, pp. 1-7, Jun. 2013.

[28] H. Z. Abidin, N. M. Din, and N. A. M. Radzi, "Deterministic static sensor node placement in wireless sensor network based on territorial predator scent marking behaviour," International Journal of Communication Networks and Information Security, vol. 5, pp. 186191, Dec. 2013.

[29] A. Peiravi, H. R. Mashhadi, and S. H. Javadi, "An optimal energyefficient clustering method in wireless sensor networks using multiobjective genetic algorithm," International Journal of Communication Systems, vol. 26, pp. 114-126, Jan. 2013.

[30] K. S. S. Rani and N. Devarajan, "Optimization model for sensor node deployment," European Journal of Scientific Research, vol. 70, pp. 491-498, Feb. 2012.

[31] Y. Mo, J. Liu, B. Wang, and Q. M. J. Wu, "A novel swarm intelligence algorithm and its application in solving wireless sensor networks coverage problems," Journal of Networks, vol. 7, pp. 20372043, Jan. 2012

[32] H. Yuan, C. Li, and M. Du, "Optimal distribution of nodes in wireless sensor network based on multi-objective optimization," Journal of Computational Information Systems, vol. 8, pp. 33313338, 2012.

[33] P. L. Matencio, “An ACOR-based multi-objective WSN deployment example for lunar surveying," Sensors, vol. 16, pp. 1-20, Feb. 2016.

[34] H. Z. Abidin, N. M. Din, I. M. Yassin, H. A. Omar, N. A. M. Radzi, and S. K. Sadon, "Sensor node placement in wireless sensor network using multi-objective territorial predator scent marking algorithm," Arabian Journal for Science and Engineering, vol. 39, pp. 63176325, Aug. 2014.

[35] A. Rajeswari and P. T. Kalaivaani, "A bi-directional energy splitable model for energy optimization in wireless sensor networks," International Journal on Advanced Science, Engineering and Information Technology, vol. 1, pp. 347-350, Jan. 2011.

[36] T. V. Chien, H. N. Chan, and T. N. Huu, "A comparative study on hardware platforms for wireless sensor networks," International Journal on Advanced Science, Engineering and Information Technology, vol. 2, pp. 70-74, 2012 